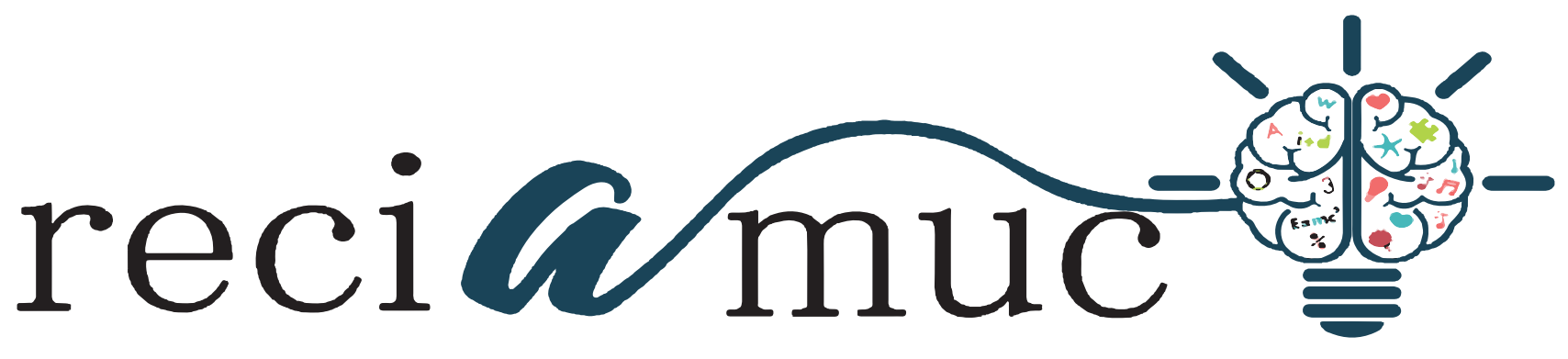

DOI: 10.26820/reciamuc/5.(3).agosto.2021.208-221

URL: https://reciamuc.com/index.php/RECIAMUC/article/view/690

EDITORIAL: Saberes del Conocimiento

REVISTA: RECIAMUC

ISSN: 2588-0748

TIPO DE INVESTIGACIÓN: Artículo de Investigación CÓDIGO UNESCO: 5801 Teoría y Métodos Educativos

PAGINAS: 208-221

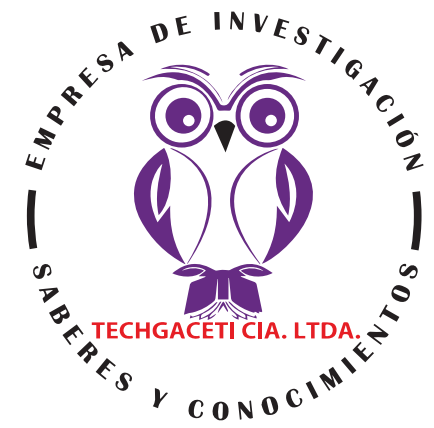

\title{
Estilos de crianza en el desarrollo de la identidad y autonomía en niños de 4 a 5 años
}

Parenting styles in the development of identity and autonomy in children from 4 to 5 years old

Estilos parentais no desenvolvimento da identidade e autonomia em crianças dos 4 aos 5 anos

Nelly Patricia Yanchapaxi Sánchezi; Gisella Antonia Solórzano Arellano2;

Victoria María Márquez Allauca3; César Raúl Molina Yanchapaxi ${ }^{4}$

\section{RECIBIDO: 10/06/2021 ACEPTADO: 12/07/2021 PUBLICADO: 31/08/2021}

1. Magister en Diseño Curricular; Psicóloga en Rehabilitación Educativa; Docente de la Universidad de Guayaquil, Facultad de Filosofía; Guayaquil, Ecuador; nelly.yanchapaxis@ug.edu.ec - nellyshina_2364@hotmail.com; https://orcid.org/00000003-3717-6600

2. Profesora en Ciencias de la Educación Especialización Educadores de Párvulos de la Universidad de Guayaquil; Ecuador.

3. Magister en Terapia Familiar Sistémica y de Pareja; Diploma Superior en Terapia Familiar Sistémica; Psicóloga Clínica; Universidad de Guayaquil; Ecuador; victoria.marqueza@ug.edu.ec; https://orcid.org/0000-0001-9093-551X

4. Médico de la Universidad de Guayaquil; Investigador Independiente; Ecuador; cesarrmy@gmail.com; https://orcid. org/0000-0002-7034-0006

\section{CORRESPONDENCIA \\ Nelly Patricia Yanchapaxi Sánchez \\ nelly.yanchapaxis@ug.edu.ec}

Guayaquil, Ecuador 


\section{RESUMEN}

Este trabajo de investigación tiene como objetivo principal determinar la influencia de los estilos de crianza en el desarrollo de la identidad y autonomía en los niños de 4 a 5 años de la Escuela de Educación Básica "Iliniza Sur" mediante una investigación bibliográfica, descriptiva, exploratoria y explicativa; con una muestra relevante para su proceso indagatorio. El estudio tiene un diseño cualitativo- cuantitativo, al realizar entrevistas a la directora de la escuela, por medios digitales debido a la emergencia sanitaria internacional en la actualidad, de la misma manera se aplicaran métodos, técnicas e instrumentos necesarios para la recolección de la información del problema, como la encuesta a los padres de familia del nivel inicial, para luego establecer las conclusiones y recomendaciones. Con el diseño de escuela para padres, se utilizaran de manera significativa estrategias motivadoras e innovadoras que generen un aprendizaje en el ámbito de Identidad y autonomía.

Palabras clave: Estilos de crianza, convivencia, escuela, Identidad y autonomía.

\section{ABSTRACT}

The main objective of this research work is to determine the influence of parenting styles on the development of identity and autonomy in children aged 4 to 5 years of the School of Basic Education "Iliniza Sur" through a bibliographic, descriptive, exploratory and explanatory research; with a sample relevant to your inquiry process. The study has a qualitative-quantitative design, when conducting interviews with the director of the school, by digital means due to the international health emergency at present, in the same way and apply methods, techniques and instruments necessary for the collection of information on the problem, such as the survey of parents of the initial level, and then establish the conclusions and recommendations. With the design of a school for parents, motivating and innovative strategies that generate learning in the field of Identity and autonomy will be used in a significant way.

Keywords: Parenting styles, coexistence, school, Identity and autonomy.

\section{RESUMO}

O objetivo principal deste trabalho de pesquisa é verificar a influência dos estilos parentais e sua vinculação com o campo da identidade e da autonomia em crianças de 4 a 5 anos da Escola de Educação Básica "lliniza Sur" por meio de uma pesquisa bibliográfica descritiva, exploratória e explicativa. ; com uma amostra relevante para o seu processo investigativo. O estudo tem um desenho qualitativo-quantitativo, ao realizar entrevistas com o diretor da escola, por meio digital devido à atual emergência sanitária, da mesma forma e serão aplicados o método, técnicas e instrumentos necessários para a coleta de informações do Caso, como é o caso do inquérito aos pais do nível inicial, para posteriormente estabelecer as conclusões e recomendações, é realizado o desenho da escola para os pais, onde estratégias motivadoras e inovadoras que geram aprendizagens no domínio da Identidade e da autonomia.

Palavras-chave: Estilos parentais, convivência, escola, identidade e autonomia. 


\section{Introducción}

Los estilos de crianza a nivel mundial son considerados patrones de conducta, importantes en el rol que desempeñan las familias porque favorecen en la formación integral de los niños, estos se encuentran en constante evolución debido a la interacción social que están sujetos. Los padres son los primeros maestros en base al estilo de crianza que se fomente en su entorno familiar, a medida del desarrollo de sus capacidades, podrá realizar actividades sin ayuda de los demás, se propone establecer parámetros innovadores a los padres de familia con el fin de lograr obtener comportamientos favorables en los establecimientos educativos.

Al reconocer que educar a un individuo es una tarea conjunta entre los docentes y los padres, se recomienda en los países desarrollados que los progenitores se esfuercen por dar tiempo a sus hijos, que logren el progreso de sus destrezas básicas vinculadas a su estilo de crianza acorde a sus leyes, sin la vulneración de sus derechos. Cabe recalcar que un niño logre su Identidad y autonomía a los 4 años de edad no es fácil, tiene su proceso, en el cual los ámbitos educativos se relacionan al estimular sus habilidades por medio de las experiencias de su vida cotidiana.

En la actualidad se observa en diversos países del mundo, el desafío de la problemática en los estilos de crianza que proporcionan los padres a sus hijos desde la infancia, sin embargo se aprecia la falta de interés en los hogares, los padres se centran en sus trabajos dejando a sus hijos en guarderías e instituciones educativas, por lo que como parte de este proceso se determina que los niños no son independientes y a su vez están desarrollando una conducta inadecuada para forjar su personalidad, si mismo se considera imprescindible la guía de sus padres para la estimulación de habilidades, cognitivas, motrices, afectivas, sociales entre otras.
Por esta razón la UNESCO (2019) establece que "Los estilos de crianza son procesos complejos que se viven en cada familia al intercambiar experiencias, información y esfuerzo de los adultos para influir significativamente en la vida de los niños y niñas". (p.115). En un estudio se comprueba que alrededor de 10 millones de niños en el mundo entero sufren de abandono por parte de sus padres. Por lo que no logran desarrollar principios para la formación socio-afectiva de la misma manera influye negativamente en el progreso del ámbito de Identidad y autonomía.

Es importante señalar que los estilos de crianza en España, son un apego ya que es donde los niños demuestran sus primeras experiencias en el fortalecimiento de su identidad y la autonomía; como lo indica (González, 2017) en su libro Estilo de crianza: Una revisión teórica hace referencia al progreso de su conocimiento, al conseguir la capacidad de resolver problemas por su propia cuenta, a su vez estas experiencias deben ser reforzadas por sus padres cada día, para que puedan acceder al desarrollo y comprensión de nuevas ideas, al estar dispuestos a enfrentar nuevos aprendizajes.

Los estilos de crianza son patrones del entorno familiar, al ser la base de la sociedad, es la encargada de formar al niño desde el vientre materno de su madre, quien estimula ese desarrollo cognitivo al hablarle, cantarle y conversar con él en todo tiempo, ella es la guía en el proceso de su crianza, de esta manera ayuda a fortalecer su identidad y autonomía al superar las dificultades que se le presenten en su vida cotidiana así estimula su autoimagen y le permite adquirir un nuevo conocimiento, lo que se percibe en Latinoamérica es una sobreprotección o vulneración de los derechos de los infantes.

Los estilos de crianza son estándares del entorno familiar, al ser la base de la sociedad, es la encargada de formar al niño desde el vientre materno es su madre, quien estimula ese desarrollo cognitivo al hablar- 
le, cantarle, conversar con sus hijos en todo tiempo lo guía en el proceso de su crianza, de esta manera ayuda a fortalecer su identidad y autonomía, al superar las dificultades que se le presenten en su vida cotidiana así estimula su autoimagen y le permite adquirir un nuevo conocimiento, lo que se percibe en Latinoamérica es una sobreprotección o vulneración de los derechos de los infantes.

En Latinoamérica al indagar sobre los continuos comportamientos de los niños en edad escolar se analiza el entorno familiar, como lo expone (Sánchez, 2017) "La familia es considerada como un organismo básico en la educación y crianza del niño, históricamente se le ha asignado funciones elementales, a partir de la idea de reproducción biológica, económica y sociocultural" (p.89). Es decir que los estilos de crianza inciden en el desarrollo potencial de los niños en los ámbitos de identidad y autonomía que son primordiales el desarrollo en la infancia.

Con el paso de los años, la manera de corregir de algunos padres o cuidadores está basado en diferentes modelos que favorecen o perjudican el desarrollo integral de sus hijos. Según (Varela, 2019), "Los enfoques pedagógicos del siglo XX Ilevaron a cuestionar la coerción y el castigo físico como métodos educativos". Es decir que los estilos de crianza han sufrido muchos cambios y sobre todo han creado influencia negativa en el desarrollo emocional de los niños suscitándose en varios países, por lo que en Paraguay fue creada la Ley $\mathrm{N} .{ }^{\circ}$ 5.659 donde se indica lo siguiente:

"Prohíbe el castigo físico y los tratos humillantes infligidos a niños, niñas y adolescentes como forma de corrección o disciplina, por parte de los padres, tutores, guardadores o responsables de su educación, cuidado, orientación, o tratamiento de cualquier clase. Establece ámbito y autoridades de aplicación de la ley" (Sistema de Información y tendencias educativas en América Latina, 2016, pág. 102).
El Ministerio de Educación del Ecuador comprometido con la necesidad de brindad una mejor calidad en el nivel inicial promueve el diseño de un currículo que garantice el desarrollo óptimo de los procesos de enseñanza aprendizaje, al reconocer en el Plan Decenal del Buen Vivir, 2017; las políticas de la primera infancia en su desarrollo integral, al incorporar bases teóricos de los estudios realizaos por Bruner, Vygotsky, Piaget, Álvarez, Tinajero entre otros, que se enfocan en las experiencias de aprendizaje desde los primeros años de vida que contribuyen en el desarrollo de la identidad y autonomía de los niños y niñas.

Es por ello que el Currículo de educación inicial propuesto por el Ministerio de Educación del Ecuador en el 2016, según el Acuerdo ministerial (Mineduc, Acuerdo Ministrial, 2018); establece las consignas de ingreso al nivel de educación inicial, el cual no es escolarizado, con el propósito de desarrollar los aspectos: psicomotriz, psicosocial, cognitivo, afectivo, de Identidad y autonomía. De acuerdo con un estudio realizado por la (Unicef, 2019), "En Ecuador ni la cuarta parte de los niños de 3 a 5 años concurren en el sistema de educación inicial, lo que se muestra en el informe detallado por de la ODS".

En la Escuela de Educación Básica Particular "Iliniza Sur", ubicada en la Provincia del Guayas, Zona 8, Cantón Guayaquil, en el Guasmo Central, Cooperativa Flor del Guasmo Manzana 4, Solar 6; se evidencia la falta de identidad y autonomía de los niños de 4 a 5 años. Al momento de tomar agua, saltar, correr, lanzar, emitir un comentario o idea, falta de creatividad, de cooperación, al no poder mencionar su nombre, entre otras destrezas que a su edad ya deberían ser adquiridas. Esta problemática es percibida por las autoridades de la institución al observar las clases demostrativas.

Es por ello que el presente trabajo de investigación con el tema de los estilos de crianza vinculados al ámbito de identidad

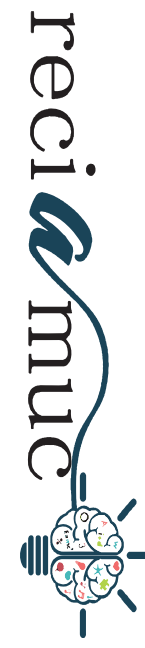


y autonomía en niños de 4 a 5 años, propone el diseño de una escuela para padres, ya que se considera que la formación integral de un niño se logra si trabajamos en conjuntos con los padres, lo que permitirá romper obstáculos que se presentan en el desarrollo de las actividades en el nivel de educación inicial a su vez emprender nuevas expectativas en el interaprendizaje del ámbito de identidad y autonomía.

\section{Metodología}

La investigación se enfoca en el ámbito educativo al determinar la influencia de los estilos de crianza en el desarrollo de la identidad y autonomía en los niños de 4 a 5 años de la Escuela de Educación Básica "Iliniza Sur" mediante una investigación bibliográfica para el diseño de escuela para padres. Se basa en un diseño cualitativocuantitativo, al realizar encuestas por medios digitales debido a la emergencia sanitaria internacional, de la misma manera y aplicaran método, técnicas e instrumentos necesarios para la recolección de la información del problema planteado.

Se establece que el presente diseño de la indagación constituye en un procedimiento general de la información al incorporar diversos tipos de investigación bibliográfica, experimental, descriptiva; a su vez los métodos de análisis, síntesis, deductivo e inductivo; para así poder obtener los resultados precisos a las interrogantes planteadas, por lo que se aplican las encuestas a padres de familia, la entrevista a la directora y la observación del contexto de forma virtual debido a la emergencia sanitaria internacional, por el virus Covid-19.

Una vez seleccionado el contexto donde se percibió la problemática en la Escuela de Educación Básica "lliniza Sur", al indagar sobre las causas del mismo, se emplea el método de investigación cualitativa al proceder a explicar, predecir, descubrir la información de manera sistemática, se genera principalmente una comprensión de las opiniones planteadas en la entrevista por medio de plataformas digitales, al centrarse en obtener datos reales de fácil comprensión de las acciones de campo en las experiencias en el desarrollo del ámbito de identidad y autonomía de los niños de 4 a 5 años.

En la recolección de la información se estableció que la investigación cuantitativa es factible al asignar valores, es decir la estadística de las tablas y gráficos que se ejecutan en la recolección de datos, al comparar los resultados obtenidos por medio de las encuestas a los padres de familia, docentes y directivo, proporcionan cifras que facilitan el desarrollo del presente proyecto en beneficio a los niños y niñas de 4 a 5 años mediante el diseño de una escuela para padres.

Este proyecto investigativo parte de cada elemento que incurre en el problema de estudio planteado y la relación que existe entre ambas variables, para determinar las causas, de la misma manera los efectos en el desarrollo de la identidad y autonomía, al consistir en la separación de cada una de sus partes al describir los contenidos en el marco teórico además se establece una base científica significativa al deducir estrategias metodológicas esenciales en el diseño de escuela para padres vinculada a los estilos de crianza.

\section{Entrevista:}

La entrevista es dirigida a la directora Lcda. María Fernanda Quimí de Llánez de la Escuela de Educación Inicial Particular "Iliniza Sur"; la misma que se aplica a través de un cuestionario de preguntas abiertas con la finalidad de reconocer el problema de estudio de la investigación, manifestada por la falta de desarrollo en el ámbito de identidad y autonomía en los niños de 4 a 5 años, por las disposiciones de las autoridades esta entrevista se realizó mediante video-Ilamada. 


\section{Encuesta:}

Es un instrumento que nos facilita conseguir la información sobre la población en el estudio propuesto de la investigación, ya que primeramente se selecciona el personal que participará, luego se realizan las preguntas mediante el diseño de un cuestionario anónimo, se procede a recolectar la información, posteriormente se analicen los datos obtenidos, por lo que se estructuro 10 preguntas para padres y directora.

\section{Cuestionario:}

Los cuestionarios son importantes en el desarrollo de la investigación, al considerarse como una herramienta clave en el proceso sistemático que facilita observar las causas del problema de estudio, al ser planteado para poder realizar los análisis estadísticos de cada una de las respuestas obtenidas de la población, del mismo modo poder clasificar y descubrir la interpretación de los resultados.

\section{Ficha de observacion}

Una ficha de observación es un instrumento significativo en el desarrollo de la presente investigación, con una estructura sencilla y fácil de completar mediante la percepción del análisis de los resultados obtenidos, con la finalidad de determinar la influencia de los estilos de crianza en el desarrollo de la identidad y autonomía de los niños de 4 a 5 años de la Escuela de Educación Básica "lliniza Sur", este proceso se realizó de manera virtual debido a la emergencia sanitaria internacional. Se detalla a continuación la ficha de observación a la docente de Inicial.

La población está compuesta por una directora, 8 docentes y 210 padres de familia de la Escuela de Educación Básica "Iliniza Sur".

Tabla 1. Población de la Escuela de Educación Básica "Iliniza Sur".

\begin{tabular}{|c|c|c|c|}
\hline Ítem & Estratos & Frecuencias & Porcentajes \\
\hline $\mathbf{1}$ & AUTORIDADES & 1 & $0,46 \%$ \\
\hline $\mathbf{2}$ & DOCENTES & 8 & $3,65 \%$ \\
\hline $\mathbf{3}$ & PADRES DE FAMILIA & 210 & $65,70 \%$ \\
\hline $\mathbf{4}$ & NIÑOS & 34 & $30,15 \%$ \\
\hline & Total & $\mathbf{2 4 4}$ & $\mathbf{1 0 0 \%}$ \\
\hline
\end{tabular}

Fuente: Secretaría del Plantel

Elaborado por: Gisella Antonia Solórzano Arellano. 
Tabla 2. Muestra de la Escuela de Educación Básica "lliniza Sur".

\begin{tabular}{|c|c|c|c|}
\hline Ítem & Estratos & Frecuencias & Porcentajes \\
\hline $\mathbf{1}$ & AUTORIDADES & 1 & $2,33 \%$ \\
\hline $\mathbf{2}$ & DOCENTES & 8 & $18,60 \%$ \\
\hline $\mathbf{3}$ & PADRES DE FAMILIA & 34 & $59,07 \%$ \\
\hline $\mathbf{4}$ & NIÑOS & 17 & $20,00 \%$ \\
\hline & Total & 60 & $100 \%$ \\
\hline
\end{tabular}

Fuente: Secretaría del Plantel

Elaborado por: Gisella Antonia Solórzano Arellano.

\section{Resultados}

Análisis e interpretación de los resultados de la encuesta aplicada a los padres de familia de la Escuela de Educación Básica "Iliniza Sur".

El ambiente familiar influye significativamente en el desarrollo de destrezas en los niños, mediante las relaciones interpersonales en su entorno determina los valores, actitudes, personalidad, afectos, decisiones que se reflejan al inicio de su etapa escolar, en las encuestas se manifiesta que en su mayoría se encuentran entre sus progenitores es decir con su madre y padre, una minoría con otros familiares o solo con el padre, por diversos motivos se desintegran ya sea por pérdida de vidas por la pandemia que se está promoviendo a nivel nacional e internacional.

Encuesta aplicada a los padres de familia de la Escuela de Educación Básica "Iliniza Sur".

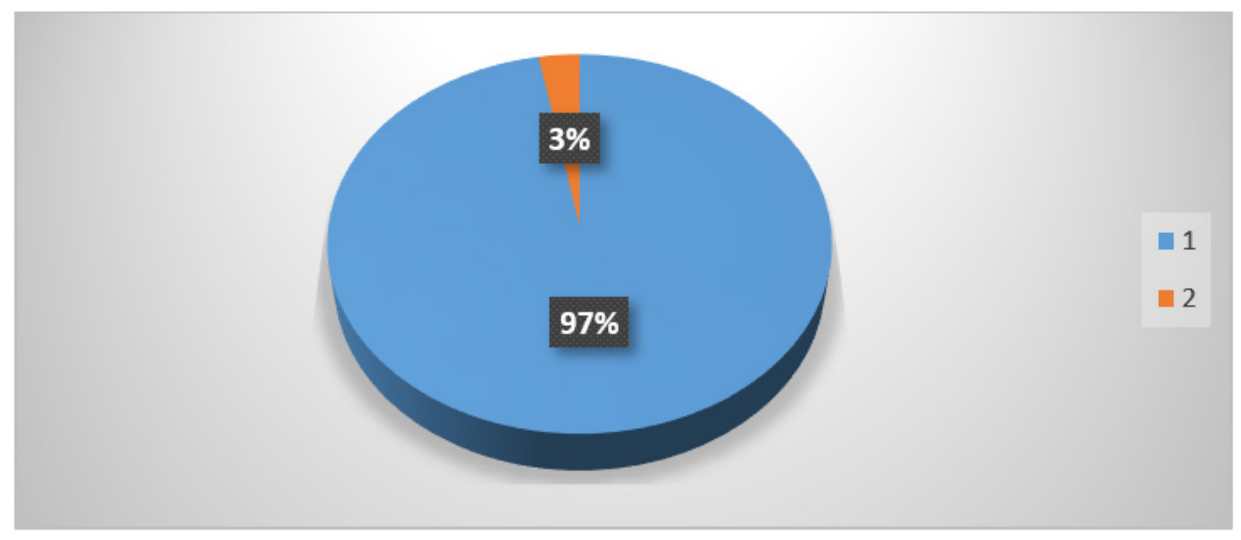

Gráfico 1. Los estilos de crianza

Fuente: Secretaría del Plantel

Elaborado por: Gisella Antonia Solórzano Arellano. 
Se percibe que los padres de familia encuestados consideran en su mayoría, no conocen de que se trata los estilos de crianza que se deben dar en el hogar, en su minoría indica que sí; es por ello que se debe realizar de urgencia una escuela para padres que proporcione información sobre este tema.

\section{¿Qué actitud asume ante un mal comportamiento de su hijo?}

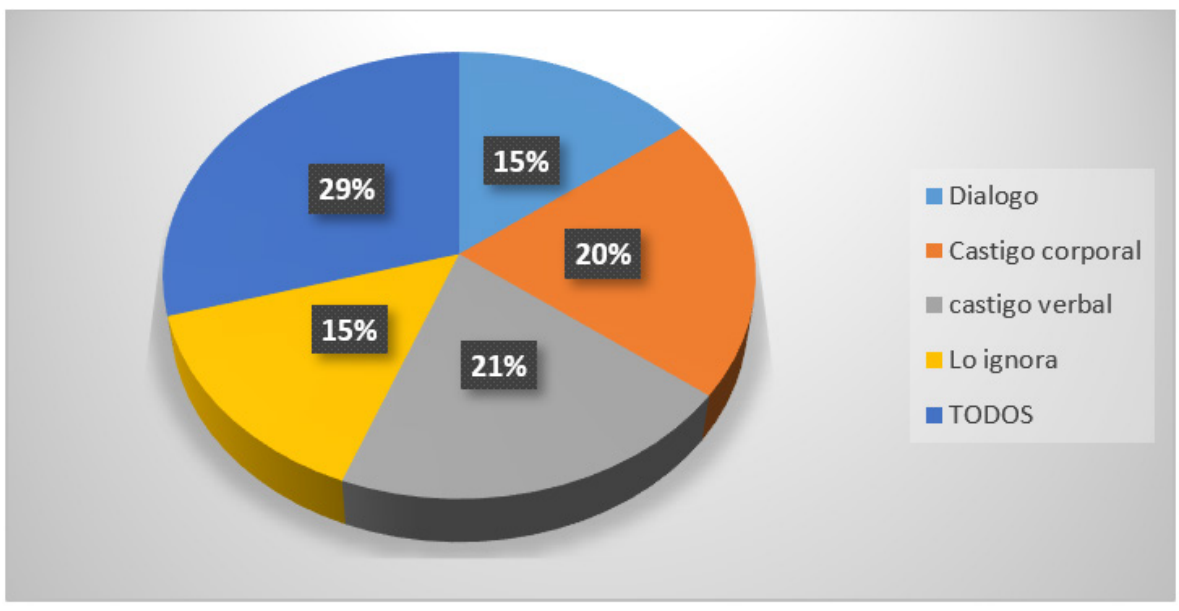

Gráfico 2. Actitud ante un mal comportamiento

Fuente: Secretaría del Plantel

Elaborado por: Gisella Antonia Solórzano Arellano.

Se observa que en su mayoría indican que todas las opciones asumen ante un mal comportamiento de su hijo, ya que en circunstancias no saben cómo actuar ante estas dificultades no hay un libro que enseñe a ser excelentes padres, pero con las experiencias se logrará motivar a perfeccionar su comportamiento.

¿Cree Ud. que es necesario utilizar los castigos?

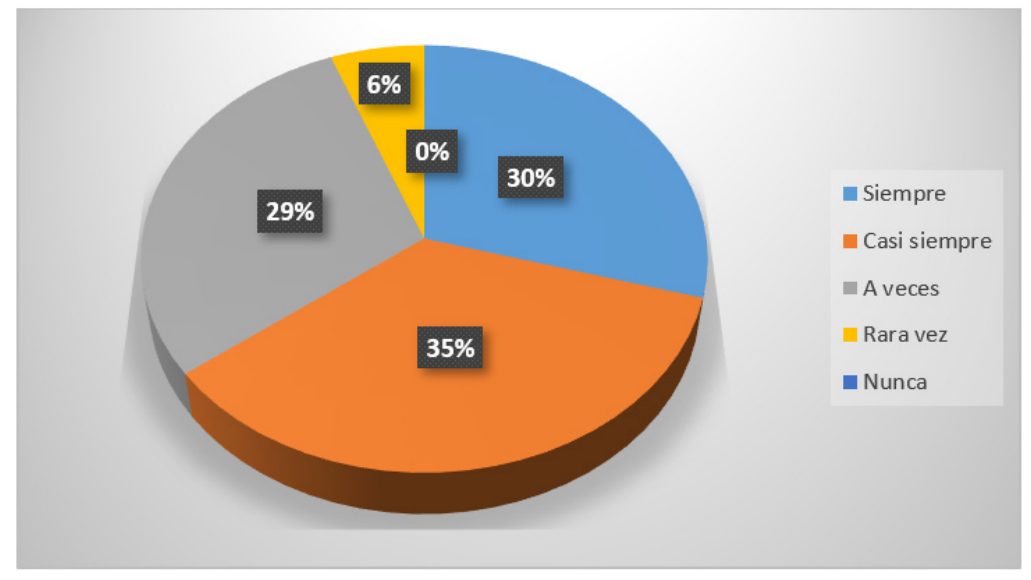

Gráfico 3. Modificaciones en el comportamiento

Fuente: Secretaría del Plantel

Elaborado por: Gisella Antonia Solórzano Arellano. 
Se evidencia en su mayoria que casi siempre es necesario utilizar menos los castigos para mejorar el comportamiento de su hijo, aunque a veces desconocen las estrategias que podrían utilizar en vez de castigarlos.

¿Cree usted que los conflictos en el hogar influyen en el comportamiento de su hijo?

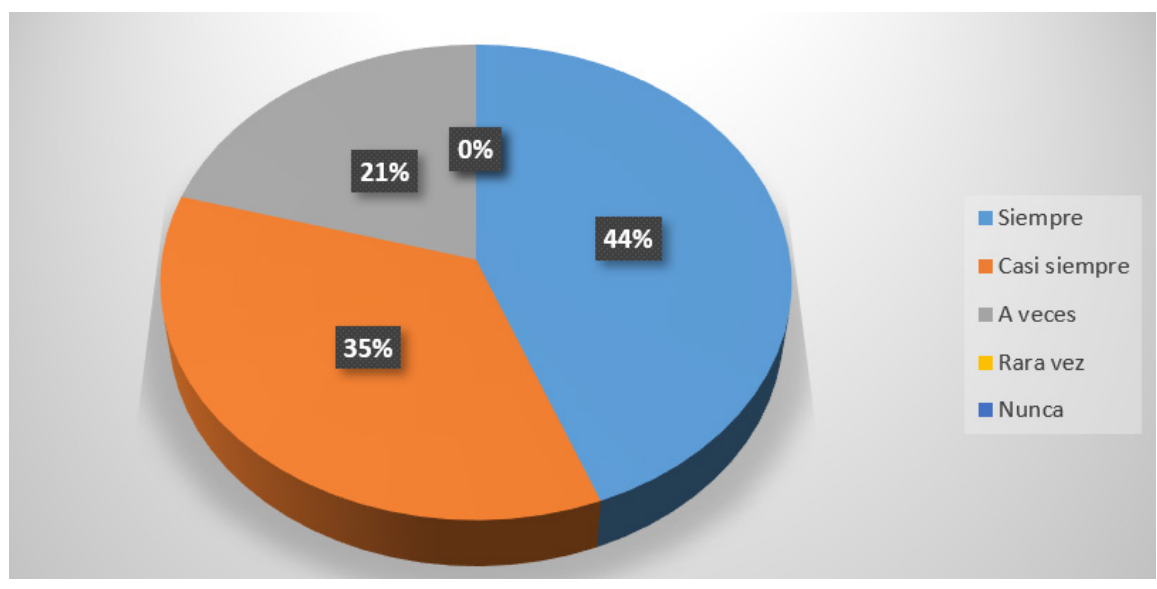

Gráfico 4. Los conflictos en el hogar influyen en el comportamiento

Fuente: Secretaría del Plantel

Elaborado por: Gisella Antonia Solórzano Arellano.

Los padres de familia indican en su mayoría que los conflictos que se suscitan en el hogar influyen en el comportamiento de su hijo, ya que ellos reflejan en sus acciones lo que ven en su entorno familiar.

\section{¿Cree Ud. que un entorno agresivo afecta en el comportamiento de su hijo?}

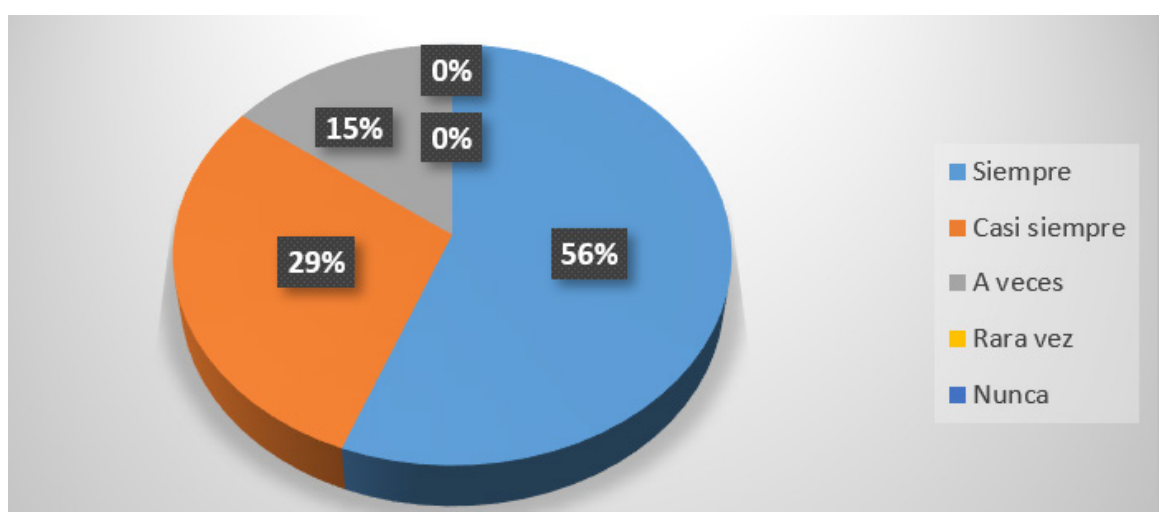

Gráfico 5. Entorno agresivo

Fuente: Secretaría del Plantel

Elaborado por: Gisella Antonia Solórzano Arellano.

Demuestran los padres de familia en su mayoría que siempre un entorno agresivo influye en el proceso educativo de un niño, ya que en algunos casos los niños cuando son muy castigados en el hogar se distraen o son muy tímidos y no participan en las actividades escolares.

¿Los estímulos afectivos que reciben los niños lograrán mejorar las relaciones interpersonales? 


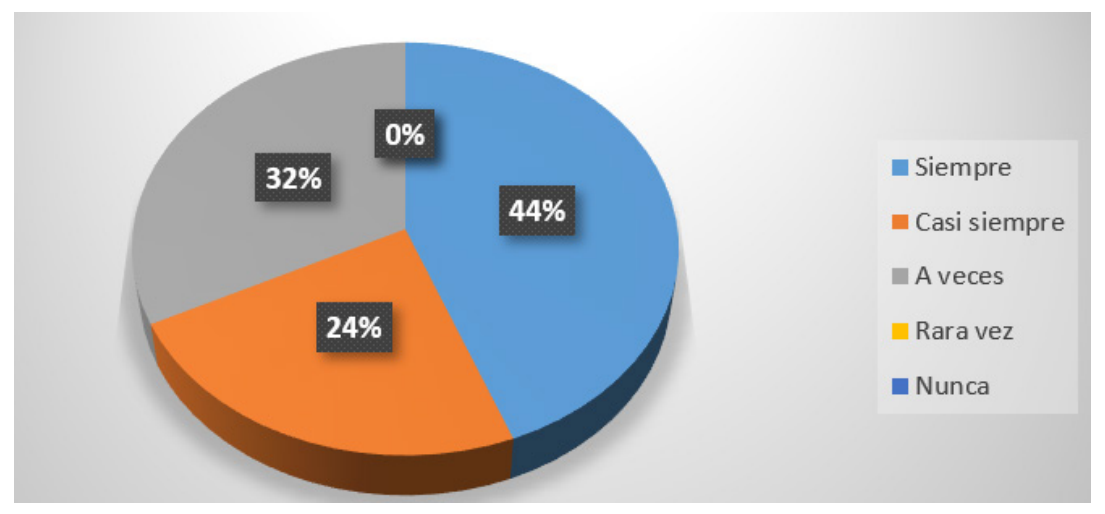

Gráfico 6. Estímulos afectivos

Fuente: Secretaría del Plantel

Elaborado por: Gisella Antonia Solórzano Arellano.

Los padres de familia en su mayoría que siempre es necesario que los niños se reúnan en familia y reciban estímulos afectivos de parte de los padres para una sana convivencia lo que influye en su comportamiento, rendimiento escolar y a su vez el fortalecimiento de su autoestima.

¿Práctica a diario muestras de afecto en su entorno familiar?

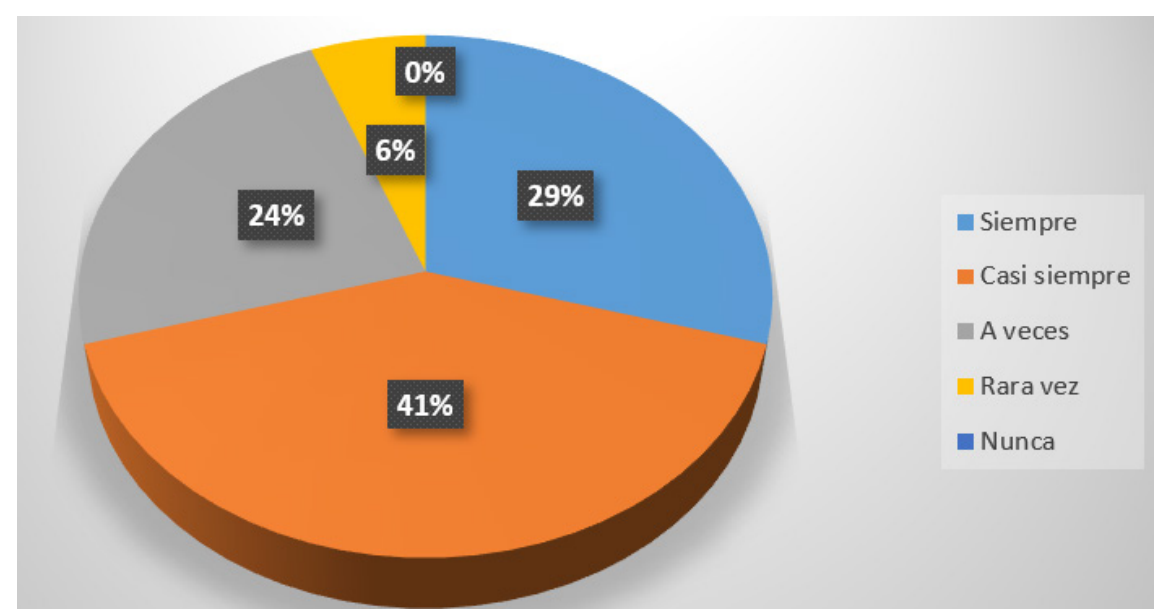

Gráfico 7. Desarrollo personal y social

Fuente: Secretaría del Plantel

Elaborado por: Gisella Antonia Solórzano Arellano.

Los padres de familia en su mayoría que casi siempre demuestra afecto a diario a sus hijos para que muestre un buen desarrollo personal y social, así mismo respetar sus opiniones y entender sus intereses.

¿Cuál es el estilo de crianza que usted cree más adecuado para la crianza de su hijo?

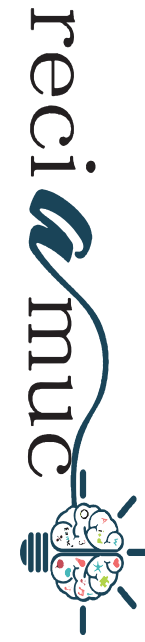




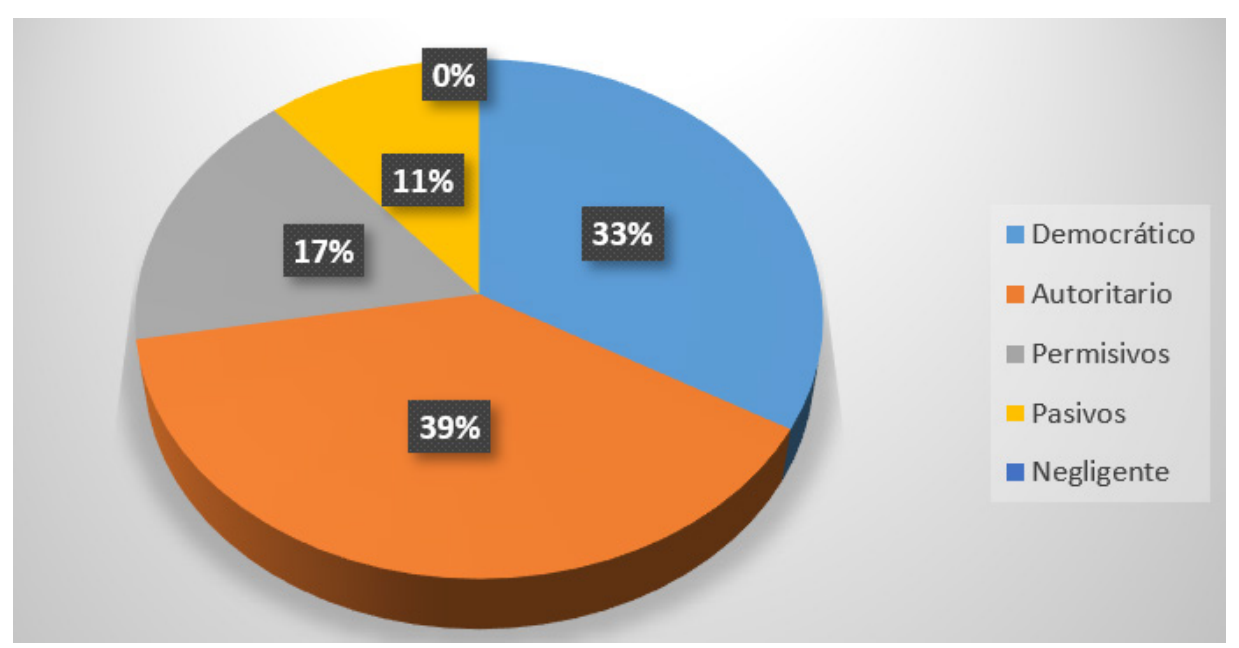

Gráfico 8. Estilo de crianza adecuado

Fuente: Secretaría del Plantel

Elaborado por: Gisella Antonia Solórzano Arellano.

Los padres de familia indican en su mayoría que casi siempre se puede aplicar un estilo de crianza adecuado para ayudar a su hijo en el proceso de aprendizaje así mismo en el desarrollo de destrezas y habilidades desde temprana edad.

¿Estaría dispuesto a participar en un taller de capacitación para aplicarlo en su hogar?

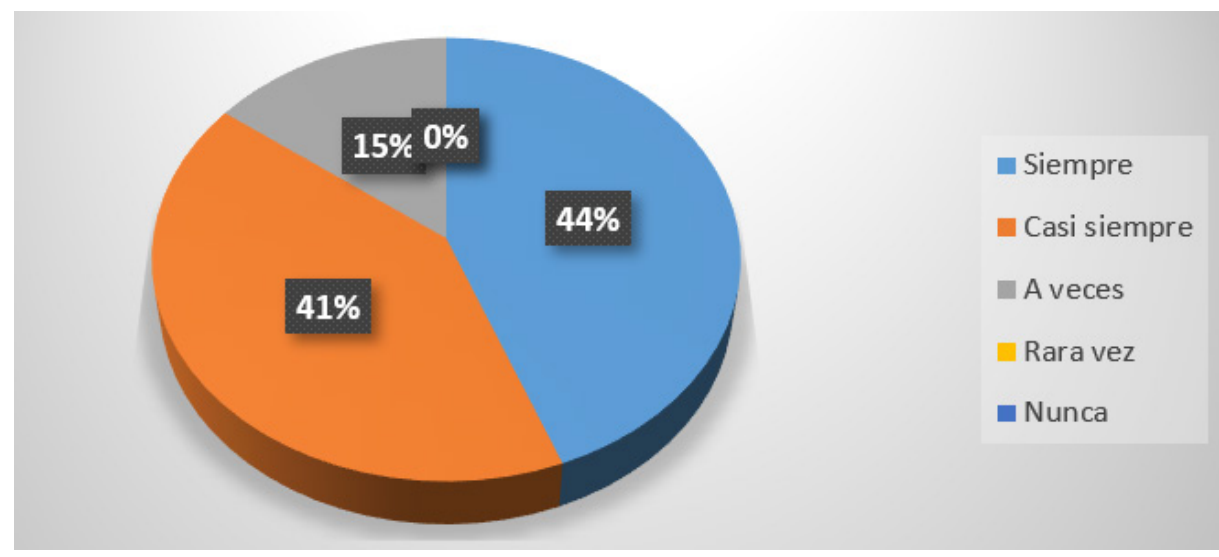

Gráfico 9. Taller de capacitación sobre los estilos de crianza

Fuente: Secretaría del Plantel

Elaborado por: Gisella Antonia Solórzano Arellano.

Se percibe que la mayoría de los padres considera importante que los padres reciban un taller de capacitación sobre los estilos de crianza para aplicarlos en el hogar y ayudar en el proceso educativo de su hijo.

¿Usted considera que algunos comportamientos impropios dentro del salón de clases afectan a la identidad y autonomía de los niños? 


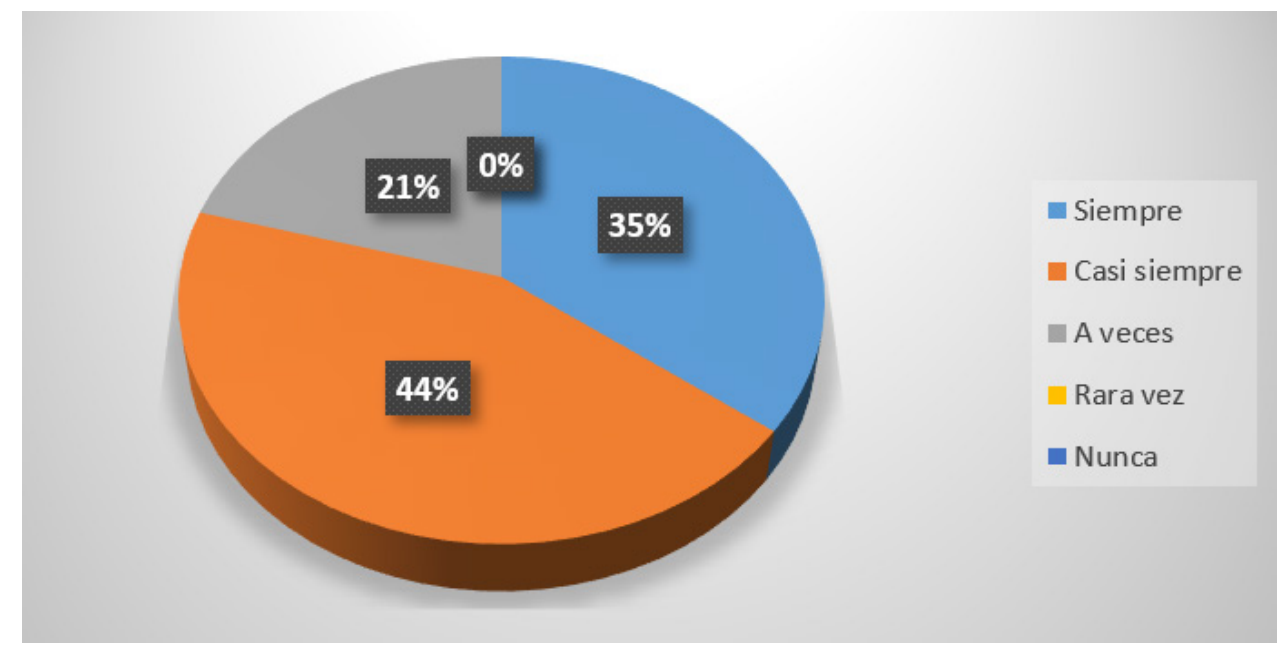

Gráfico 10. Ambiente escolar

Fuente: Secretaría del Plantel

Elaborado por: Gisella Antonia Solórzano Arellano.

Se percibe que en su mayoría los padres de familia consideran que algunos comportamientos de los niños afectan en el ambiente escolar es por ellos se debe diseñar estrategias idóneas en el proceso de los estilos de crianza en beneficio al desarrollo de las habilidades y destrezas en el ámbito de identidad y autonomía de los niños de 4 a 5 años de la Escuela de Educación Básica "lliniza Sur".

\section{Conclusiones}

Al concluir el análisis e interpretación de los resultados obtenidos mediante los instrumentos empleados en la investigación, al entrevistar a la directora de la Escuela de Educación Básica Particular "Iliniza Sur", Lcda. María Fernanda Quimí de Llánez, así mismo las encuestas realizadas a los padres de familia se enfocan en que los progenitores no le dan la debida importancia a los estilos de crianza que están que aplican en el hogar.

os estilos de crianza guardan relación con el desarrollo de la Identidad y autonomía, cuando los niños demuestran una falta de interés en el proceso de aprendizaje, se muestran sumisos, agresivos, desobedientes, entre otros aspectos ya que la forma- ción de los padres construye fundamentalmente el comportamiento de los niños en el desarrollo de su identidad y autonomía.

Los estilos de crianza se caracterizan por un patrón de comportamiento repetitivo es decir como los padres influyen en el desarrollo de la identidad y autonomía de sus hijos, que desde temprana edad demuestran su personalidad, destrezas y habilidades en la resolución de problemas de la vida diaria.

Los docentes poseen poco conocimiento sobre los estilos de crianza de sus estudiantes y por ello no comprenden su comportamiento en los salones de clases, que explica la deficiencia en el desarrollo en la identidad y autonomía.

La institución educativa Particular "Iliniza Sur", no cuenta con estrategias metodológicas de una escuela para padres para ayudar en el problema planteado en la investigación.

Se recomienda a los padres de familia de la Escuela de Educación Básica Particular "Iliniza Sur" que deben acudir a la escuela para padres que organicen la institución educativa en beneficio al desarrollo de la identidad y autonomía mediante la influen- 
cia de los estilos de crianza que otorgan sus progenitores en el hogar.

\section{Propuesta}

II. Denominación: "Escuela para los padres de familia que reconozcan los estilos de crianza que aplican en los niños de 4 a 5 años para el desarrollo del ámbito de identidad y autonomía".

¿ La "Escuela para padres" ha sido diseñado para que los progenitores o representantes legales de los niños del nivel Inicial II de la Escuela de Educación Básica Particular "Iliniza Sur" fortalezcan las relaciones interpersonales con sus representados mediante la aplicación de estrategias elementales en el proceso de aprendizaje en el ámbito de identidad y autonomía.

La "Escuela para padres" se aplicará en 8 sesiones dinámicas donde se reflejan estrategias de debate, lluvia de ideas, dramatizaciones, exposiciones, comunicación abierta al dialogo, concursos, juegos, participación activa. (Muestra de 34 padres de familia).

Primará la metodología activa y participativa en la "Escuela para padres", orientándose en el trabajo en equipo a través del instrumento lista de cotejo.

III. Estrategias metodológicas:

- Dinámicas grupales sobre los estilos de aprendizaje.

- Dramatizaciones familiares.

- Lluvia de ideas.

- Exposiciones.

- Debate.

- Proyectos de inclusión.

- Lecturas.

- Juegos didácticos.

- Participación.

- Trabajo en equipo.
- Trabajo colaborativo.

Es primordial que las actividades que se implementan en esta escuela para padres estén diseñadas para el desarrollo de la Identidad y autonomía de los niños de 4 a 5 años, con el propósito de motivar a los padres de familia a mejorar las relaciones con sus representados mediante los estilos de crianza que se aplican en el hogar, de forma que se predomine la creación de reglas para mejorar su comportamiento, la comunicación constante para incrementar el vocabulario, el juego para mantener una sana convivencia y pueda expresar sus sentimientos del mismo modo sus pensamientos.

Las estrategias que se aplican son eficientes en la labor educativa ya que si un niño de este nivel vive una hermosa etapa con sus progenitores donde puede ser independiente y autónomo en las labores diarias adquieren destrezas y habilidades en el aspecto pedagógico, didáctico, recreativo, intelectual por medio de las experiencias de aprendizaje, el padre de familia debe ser la guía en el hogar, así como en las instituciones educativas es el docente. Los padres tienen en sus manos la gran responsabilidad de educar a sus hijos en un camino correcto, con principios, así obtendremos adultos felices, independientes, capaces de luchar por sus ideales

\section{Bibliografía}

Bernal. (2016). Percepciones educativas de identidad en los infantes. Sevilla. es, 183.

González. (2017). Estilos de Crianza parental: una revisión teórica. Barcelona- España: Universidad Nacional.

Mineduc. (2018). Acuerdo Ministrial. Quito-Ecuador: 2018-00056-A.

Sánchez. (2017). Modelos de crianza. Chile: Universidad de Chile.

SITEAL. (2016). Sistema de Información y tendencias educativas en América Latina.

UNESCO. (2019). Acompañando la crianza Guía 
para el desarrollo de las sesiones de educación inicial. México: Consejo Nacional de Fomento Educativo.

Unicef. (2019). Educación Inicial. Ecuador: https:// www.elcomercio.com/actualidad/ninos-educacion-inicial-estudiantes-padres.html\#: :tex$\mathrm{t}=$ La\%20educaci\%C3\%B3n\%20inicial\%20se\%20 divide,uno $\% 20$ es $\% 203 \% 20$ a $\%$ C3\%B10s\%20 cumplidos.

Varela, S. P. (2019). Tendencias de investigaciones sobre prácticas de crianza en Latinoamerica. Infancias imagenes.

\section{CITAR ESTE ARTICULO:}

Yanchapaxi Sánchez, N. P., Solórzano Arellano, G. A., Márquez Allauca, V. M., \& Molina Yanchapaxi, C. R. (2021). Estilos de crianza en el desarrollo de la identidad y autonomía en niños de 4 a 5 años. RECIAMUC, 5(3), 208-221.

https://doi.org/10.26820/reciamuc/5.(3).agosto.2021.208-221

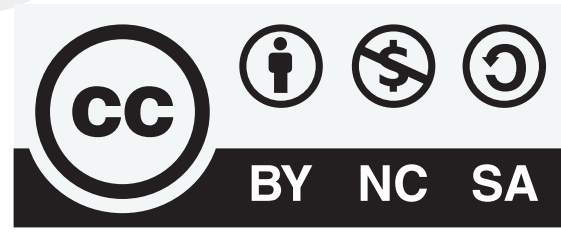

CREATIVE COMMONS RECONOCIMIENTO-NOCOMERCIAL-COMPARTIRIGUAL 4.0. 